\title{
The Role of Particle Therapy in the Risk of Radio-induced Second Tumors: A Review of the Literature
}

\author{
ANGELICA FACOETTI, AMELIA BARCELLINI, FRANCESCA VALVO and MARCO PULLIA \\ National Center of Oncological Hadrontherapy, Fondazione CNAO, Pavia, Italy
}

\begin{abstract}
One of the most important late side-effects of radiation therapy is the development of radiation-induced secondary malignancies. In the last years, this topic has significantly influenced treatment decision-making as the number of long-term cancer survivors has significantly increased with advances in treatment modalities. All efforts are being made to prevent the incidence of tumors induced by radiation. In this review article we summarize the current knowledge about treatment-related secondary cancers with a particular attention to hadrontherapy.
\end{abstract}

Radiation therapy (RT) is a central treatment modality in the treatment of cancer, with approximately $50 \%$ of all cancer patients receiving radiation therapy during their course of illness. RT contributes towards $40 \%$ of the curative treatment for cancer (1).

RT can be given with the intent of cure as well as being used as a palliative treatment to relieve patients from symptoms caused by the cancer and it is used in combination with surgery, chemotherapy or immunotherapy.

Because of the improvement in cancer survival, the longterm radio-induced toxicity is becoming an increasingly important issue as it can negatively influence the healthrelated quality of life of the survivors. In particular, radiationinduced secondary primary malignancies (SPMs) are late complications of radiotherapy, seen among the survivors of both adult and pediatric cancers. Due to their longer postexposure life expectancy, up to $80 \%$ at 5 years, the lifetime risk of developing radiation-induced malignancies (SPMs) is elevated in all childhood survivors (2).

Correspondence to: Angelica Facoetti, Fondazione CNAO, Strada Campeggi 53, Pavia, Italy. Tel: +39 0382078455, Fax: +39 0382078903, e-mail: Facoetti@cnao.it

Key Words: Hadrontherapy, second tumours, radiotherapy, cancer survivorship, review.
Radiation-induced SPMs typically arise after long latencies of a decade or more following RT and are known to have a worse prognosis than sporadic cancer because of their more aggressive biological characteristics and the higher risk of cumulative toxicities.

Epidemiological data, particularly from Japanese atomic bomb survivors, has shown that tumour development was increased in this population compared to non-irradiated individuals and even if these results are difficult to translate into clinical experience because of the very specific exposure conditions (such as overall irradiation time, dose rate, type of radiation), data confirm that at lower doses, the radiation risks are primarily stochastic effects, in particular, somatic effects (cancer) rather than the deterministic effects characteristic of higher-dose exposure.

In 1948, Cahan et al. defined the criteria for the diagnosis of radiation-induced SPMs (3) successively modified by Singh et al. in order to use them also in other histologies (4) as follows: a) SPMs must have arisen in an irradiated field, b) Between the initial irradiation and the alleged induced malignancy, a sufficient latent period, preferably longer than 4 years, must have passed, c) The two tumors must have been biopsied and be of different histology, d) The tissue in which the alleged SPMs arose must have been metabolically and genetically normal before the radiation exposure.

It is very complex to assess the risk of radio-induced SPMs because the rarity of the event and the difficulty to find a control group such as patients with the same cancers treated with or without RT (except prostate and cervix uterine cancer in which surgery could be an alternative).

\section{Pediatric Patients}

It is well accepted that the risk of developing SPMs following radiotherapy in adults is low compared to the benefits of the treatment, but this may not be so evident for pediatric tumors. It has been estimated that the risk of developing SPMs among children undergoing radiation therapy may be up to 10 times greater than in adults (5). The reasons for this increased 
susceptibility are still unclear, although it has been hypothesized that, in addition to a longer life expectancy, the damage caused to stem cells, which are more active in children's tissues than in the tissues of adults, has a key role. In children, it is also important to take into account hereditary mutations, lifestyle factors and/or environmental factors that may have caused the onset of the first tumor. Last but not least, the small body size of pediatric patients makes the volume of healthy tissue exposed to leakage radiation much bigger, especially with the use of the modern radiotherapy techniques, as reported in 2006 by Hall EJ (5). Shuryak hypothesized in his mathematical models that there may be different dominant factors underlying the variations in risk of radio-induced SPMs depending on age: the mechanisms of action of radiation as initiator and promoter would have different "weights" depending on the age of patients. In the case of pediatric exposure, the radiation would act primarily as an initiator and the "initiated" cells would have more time to show their growth advantage over normal cells. On the other hand, the normal tissues of adult patients have already accumulated numerous premalignant cells in the course of their life and on these cells the radiation would act mainly as a promoter (6).

From the epidemiological point of view, the data collected in different European and US studies confirm that the dependence on the radiation dose in the induction of SPMs is different in the various organs and tissues, also in the pediatric context. The high doses that are reached in the treatment volume or very close to it cause more often the onset of soft tissue sarcomas (which represent $50 \%$ of all secondary cancers in the entire epidemiological study), while carcinomas, in particular adenocarcinomas of the breast and thyroid occur mostly in the penumbra of the radiation field (7, 8). The Childhood Cancer Survivor Study (CCSS) published the largest retrospective study in pediatric oncology patients evaluating 14,359 patients, of whom 2,703 developed SPMs $(9,10)$. This study showed that there is a relationship between the relative risk and the dose received for the treatment of primary cancer for the onset of SPMs in the central nervous system, breast and thyroid. Gliomas were mainly observed in the high-dose regions before reaching age 30 in patients who had previously been radiotreated for brain tumors, while breast tumors were observed in low-dose regions after the age of 30. Among SPMs, meningiomas represent the most frequent tumor histology of the CNS, followed by gliomas, mostly of high degree. The excess risk for brain tumors would increase with increasing dose for both histologies and is greater for children under 5 years old. Gliomas were observed after an average of 9 years from the diagnosis of the original tumor, whereas meningiomas after 17 years. Finally, radio-induced meningiomas were often found to be multiple, in contrast to meningiomas not associated with previous radiotherapy. Their incidence is associated with young age, they show aggressive histology and peculiar cytogenetic characteristics, including $1 \mathrm{p}$ and 22q deletions (11).

\section{Photon Beam Radiation therapy}

Brenner et al. have analyzed the Surveillance, Epidemiology, and End Results Program cancer registry (SEER) database to compare secondary malignancies in prostate cancer patients who were treated with either surgery $(70,539$ patients) or RT (51,584 patients) (12). Authors showed that $50 \%$ of the radio-induced SPMs occurred in the low-dose volume (doses <1-3 Gy) and 50\% in the high-dose volume (maximum doses $>60 \mathrm{~Gy}$ ); secondary tumours have been noted in rectum, bladder, oesophagus, and lung with an increased relative risk of $15 \%$ and $34 \%$ for those who survived $\geq 5$ years and $\geq 10$ years, respectively. The majority of secondary cancers were bladder and rectal cancers. In absolute terms, the estimated risk of developing a radiationassociated secondary malignancy was 1 in 290 for all prostate carcinoma patients treated with radiotherapy, increasing to 1 in 70 for long-term survivors ( $\geq 10$ years).

The radiation dose dependence of cancer induction differs between organs and tissues, suggesting the existence of different, still unclear, mechanisms (13), even if quiescent cells require high RT doses to stimulate their proliferation.

The Brenner's study represents the larger study on the induction of secondary tumors in prostate cancer patients treated with radiotherapy compared to similar patients treated with surgery alone. However, these results are based on data collected from patients treated with old radiotherapy techniques delivering whole pelvic 60 Co irradiation. In this case, a uniform dose of $60 \mathrm{~Gy}$ in the whole pelvic area was administrated and the estimated dose to the lungs is $0.6 \mathrm{~Gy}$ justifying the high incidence of radio-induced lung carcinomas recorded in these patients.

Because of the improvement in RT technologies, these data are difficult to translate in the modern RT. In the last years, secondary cancer risk following radiotherapy is an increasingly important topic in clinical oncology with a higher impact on treatment decision making and on patient management. In the modern LINAC method using the beam collimator system to improve the target coverage with a consequently better local control, the high $\mathrm{Z}$ wedge filter into the pathway of high-energy photons could lead to an increase in the number of secondary neutrons adding up to other leakage radiations. The large number of beams typically used in modern RT, such as IMRT, causes a bath of low dose in normal tissue with a higher risk of radioinduced SPMs analyzed in several dosimetric studies (1417). These data support the advantage of proton beam RT in reducing the risk of radio-induced SPMs. In fact, protons are characterized by dosimetric properties resulting in a 
reduced entrance dose that is minimal when compared with standard photon RT. Therefore, proton beam RT enables the delivery of adequate target volume coverage, reducing integral dose delivered to surrounding tissues with a potential reduced risk of radiation-induced SPMs.

The passive modulation of proton beams determines an increase in dose at the limits of the field of irradiation by a factor of 10 compared to IMRT with X-rays. Conversely, if the proton beam is actively scanned, the greatest benefits are obtained with a dose off the target volume reduced up to ten times (5).

\section{Hadrontherapy}

Due to the short follow-up time and the low number of patients treated with hadrontherapy and in particular with carbon ions, it is not possible to draw robust conclusions compared to epidemiological studies about photons.

Dosimetric studies and mathematical models have shown that the risk of secondary malignancies was lower in hadrontherapy and the probable reason for this is that the dose deposited by particles ends sharply nears the end of their range, giving rise to the Bragg peak, reducing the dose given to normal tissues.

However, there are many uncertainties in choosing RBE values (relative biological efficacy) for high LET (linear energy transfer) radiations such as carbon ions and neutrons for the tumor induction, especially in paediatric patients.

In laboratory animals, RBE values depend on the type of radiation, time of irradiation and age and it is extremely difficult to obtain robust in vivo data keeping in consideration all the different variables such as type of particle, particle energies, type of tissues and age of the subject.

How preclinical results could be used or translated in clinical risk models is still an open question; in fact, it is well known that there are many factors, both clinical and biological (genetic, demographic, lifestyle, primary tumor, adjuvant therapies, radiotherapy protocols, exposure to environmental and biological factors), that could influence the response to radiation, in addition to the intrinsic radiosensitivity.

Few in vivo studies analysed the pattern of genetic changes induced by carbon ion irradiation. In a Japanese study, T-cell lymphomas induced by irradiation with CIRT (20 samples) and photons (12 samples) were analyzed in order to characterize the genetic changes. The mice were exposed to low LET beams $(13 \mathrm{kev} / \mu \mathrm{m})$, to simulate exposure of healthy tissues in clinical practice, and to a total dose of 4-4.8 Gy delivered either as a single dose or in four fractions, one per week. Animals were monitored: a large number of $\mathrm{T}$ cell lymphomas arose in these mice but the frequency of large interstitial deletions was higher in the CIRT group (18).
The previously mentioned EJ Hall's work on the risk of the induction of secondary malignancies by neutrons generated from the interaction of high-energy protons with matter (in the accelerator's head or in the patient's body) has stirred further research.

NCI epidemiologists rejected the work of Chung et al. criticizing their design and their conclusion of lower risk of secondary tumors after proton beam radiotherapy $(19,20)$. Although these results were criticized, they promoted discussion about the carcinogenic risk due to leakage neutrons that are a problem for both high-energy photon and charged-particle treatments. In fact, photons at energies above 8-10 MV (depending on the target material) produce neutrons via photonuclear reactions.

High energy photons provide a better dose distribution in depth and, in the treatment of deep tumors, are preferable compared to lower energy X-rays. However, high-energy photons interact with materials in collimators and the beam delivery system and can cause photonuclear reactions, which yield unwanted neutrons that contribute to the risk of secondary tumors. Photoneutrons are the major contributors at doses of $18 \mathrm{MV}$ photons and at distances $>20 \mathrm{~cm}$ from the target and, because the dose tends to flatten rather than to decrease at a greater distance, the patient is immersed in a 'neutron bath'. With passive proton beam modulation, many neutrons are produced in the treatment unit and it has been hypothesized that the leakage of these neutrons could substantially increase the risk of SPMs in distal organs. Authors explained that heavy ions also produce secondary particles by nuclear fragmentation, and these particles can deposit their energy into the normal tissue. Neutrons undergo nuclear collisions with protons in water, generating additional charged particles that can ionize surrounding molecules. Exposure to leakage neutrons can be significantly reduced by using magnetically scanned beams instead of passively scattered beams (10).

To the best of our knowledge, there is only one study which analyzes the risk of secondary tumours in prostate patients after CIRT compared to surgery and photon RT series. This retrospective study compared the data of 1455 patients treated with CIRT at the Japanese NIRS centre (follow-up: 7.9 years) to 1983 patients treated with photons (follow-up: 5.7 years) and 5948 operated patients (followup: 6.0-year). Despite the multiple limitations of this study highlighted also by the authors themselves, it emerged that patients treated with CIRT had a lower risk of developing SPMs compared to those treated with photons (21).

\section{Bystander Effects}

Following the numerous experimental evidence collected in the last two decades, low dose radiobiology has witnessed an important change of perspective, establishing that the 
biological effects of ionizing radiation are not limited to the directly hit cells, but they can also involve cells that are in proximity (through physical or chemical contact). It has been hypothesized that underlying these effects, called "bystander effects", there is the cell communication mediated by signalling molecules (cytokines, oxygen and/or nitrogen free radicals) between irradiated cells and not-irradiated ones through gap junction and/or the culture medium (22). These effects do not seem to be correlated with the dose, that is, they do not increase with increasing the radiation dose and therefore their relevance could be higher at low doses. Several bystander effects were recorded, including increased cell transformation, apoptosis induction, production of oxygen and nitrogen reactive species, chromatid exchange, micronucleus frequency, instability genomics. Because many of these effects are related to carcinogenesis, their role in estimating the risk of cancer following occupational, medical and environmental exposures may not be negligible.

In 2008, Mancuso and colleagues demonstrated in vivo, for the first time, that genetic damage from ionizing radiation can also affect organs not directly exposed to the radiation themselves and that this indirect damage is able to induce the development of tumors in unexposed tissues (23). This study was conducted using a heterozygous mouse model for the Patched gene, which causes susceptibility to develop tumors after exposure to ionizing radiation. In their experiments, the Authors observed a group of 2-days -old mice whole body irradiated with a single dose of $3 \mathrm{~Gy}$ (X rays) with the head shielded by a lead cylinder. The authors observed medulloblastoma development in $39 \%$ of irradiated mice with the head shielded. Since both experimental and modeling dosimetry studies showed that a percentage equal to $1.2 \%$ of the administered dose ( $3 \mathrm{~Gy}$ ) could reach the brain despite the shielding, the Authors, to dispel any doubt, have whole body irradiated a further group of mice with a dose of $0.036 \mathrm{~Gy}$. No tumor induction was observed in this group of animals. Subsequently, further experimental evidence on the induction of bystander effects has been accumulated following in vivo exposure of zebrafish, of various species of earthworms and also of vegetables: these effects have been proven to be species and tissue-dependent (24-26).

\section{Conclusion}

It is evident that the SPMs induced by radiotherapy and, in general by any oncological treatment, represent a particularly important problem for pediatric patients, who are intrinsically more sensitive to carcinogen-induced tumorigenesis than adults and have a longer life expectancy. From the analysis of the data collected from the experience carried out so far, with both photons and protons, it has emerged that the relative risks of cancer in children exposed to radiation for cancer treatments can be very high following standard radiotherapy schemes, especially with the modern techniques of beam passive collimation. Furthermore, it has been observed that RT treatments can induce a wide variety of tumor histologies, which cannot be distinguished from the natural tumor and that carcinomas and leukaemias are commonly observed in organs receiving low doses of radiation or are far from treatment site. Sarcomas are observed mainly in tissues or organs that receive high doses of radiation or within the treatment field (27).

Despite the high degree of uncertainty due to the few patients treated so far and above all to the short follow-up time elapsed, the data acquired up to now and the dosimetric/ modeling studies suggest that particle radiotherapy, in particular with protons dispensed with active scanning, involves a lower risk of SPMs compared to conventional photon therapies. This is mainly due to the lower doses to which healthy tissues are exposed to and the low relative risk associated with exposure to neutrons throughout the body, especially when active scanning beams are used.

\section{Conflicts of Interest}

The Authors declare that the work was conducted in the absence of any commercial or financial relationships that could be construed as a potential conflict of interest.

\section{Authors' Contributions}

$\mathrm{AF}$ and $\mathrm{AB}$ wrote the draft, $\mathrm{FV}$ and $\mathrm{MP}$ critically revised the manuscript.

\section{Acknowledgements}

This review has been partially presented by $\mathrm{AF}$ at the Italian Association for Medical Radioprotection meeting (AIRM, Pavia, May 30th- June 1st, 2019).

\section{References}

1 Kumar S: Second malignant neoplasms following radiotherapy. Int J Environ Res Public Health 9(12): 4744-4759, 2012. PMID: 23249860. DOI: 10.3390/ijerph9124744

2 Smith MA, Seibel NL, Altekruse SF, Ries LA, Melbert DL, O'Leary M, Smith FO and Reaman GH: Outcomes for children and adolescents with cancer: challenges for the twenty-first century. J Clin Oncol 28: 2625-2634, 2010. PMID: 20404250. DOI: $10.1200 / \mathrm{JCO} .2009 .27 .0421$

3 Cahan WG, Woodard HQ, Higinbotham NL, Stewart FW and Coley BL: Sarcoma arising in irradiated bone; report of 11 cases. Cancer 1: 3-29, 1948. PMID: 18867438. DOI: 10.1002/10970142(194805)1:1<3::aid-cncr2820010103>3.0.co;2-7

4 Singh GK, Yadav V, Singh P and Bhowmik KT: Radiationinduced malignancies making radiotherapy a "two-edged sword": a review of literature. World J Oncol 8: 1-6, 2017. PMID: 28983377. DOI: 10.14740/wjon996w 
5 Hall EJ: Intensity-modulated radiation therapy, protons, and the risk of second cancers. Int J Radiation Oncology Biol Phys 65: 1-7, 2006. PMID: 16618572. DOI: 10.1016/j.ijrobp.2006.01.027

6 Shuryak I, Sachs RK and Brenner DJ: Cancer risks after radiation exposure in middle age. J Natl Cancer Inst 102: 16281636, 2010. PMID: 20975037. DOI: 10.1093/jnci/djq346

7 Robison LL, Mertens AC, Boice JD, Breslow NE, Donaldson SS, Green DM, Li FP, Meadows AT, Mulvihill JJ, Neglia JP, Nesbit ME, Packer RJ, Potter JD, Sklar CA, Smith MA, Stovall M, Strong LC, Yasui Y and Zeltzer LK: Study design and cohort characteristics of the childhood cancer survivor study: a multiinstitutional collaborative project. Med Pediatr Oncol 38: 229239, 2002. PMID: 11920786.

8 Tukenova M, Guibout C, Hawkins M, Quiniou E, Mousannif A, Pacquement H, Winter D, Bridier A, Lefkopoulos D, Oberlin O, Diallo I and de Vathaire F: Radiation therapy and late mortality from second sarcoma, carcinoma, and hematological malignancies after a solid cancer in childhood. Int J Radiat Oncol Biol Phys 80: 339-348, 2011. PMID: 20646844. DOI: 10.1016/j.ijrobp.2010. 02.004

9 Neglia JP, Robison LL, Stovall M, Liu Y, Packer RJ, Hammond S, Yasui Y, Kasper CE, Mertens AC, Donaldson SS, Meadows AT and Inskip PD: New primary neoplasms of the central nervous system in survivors of childhood cancer: a report from the Childhood Cancer Survivor Study. J Natl Cancer Inst 98: 1528-1537, 2006. PMID: 17077355. DOI: 10.1093/jnci/djj411

10 Newhauser WD and Durante M. Assessing the risk of second malignancies after modern radiotherapy. Nat Rev Cancer 11: 438-448, 2011. PMID: 21593785. DOI: $10.1038 / \mathrm{nrc} 3069$

11 Braunstein $\mathrm{S}$ and Nakamura JL: Radiotherapy-induced malignancies: review of clinical features, pathobiology, and evolving approaches for mitigating risk. Front Oncol 3: 73, 2013. PMID: 23565507. DOI: 10.3389/fonc.2013.00073

12 Brenner DJ, Curtis RE, Hall EJ and Ron E: Second malignancies in prostate carcinoma patients after radiotherapy compared with surgery. Cancer 88: 398-406, 2000. PMID: 10640974. DOI: 10.1002/(sici)1097-0142(20000115)88:2<398::aid-cncr22>3.0. $\mathrm{co} ; 2-\mathrm{v}$

13 Trott KR: Special radiobiological features of second cancer risk after particle radiotherapy. Physica Medica 42: 221-227, 2017. PMID: 29103987. DOI: 10.1016/j.ejmp.2017.05.002

14 Followill D, Geis P and Boyer A: Estimates of whole-body dose equivalent produced by beam intensity modulated conformal therapy. Int J Radiat Oncol Biol Phys 38: 667-672, 1997. PMID: 9231693. DOI: 10.1016/j.ejmp.2017.05.002

15 Verellen D and Vanhavere F: Risk assessment of radiation induced malignancies based on whole-body dose equivalent estimates for IMRT in the head and neck region. Radiother Oncol 53: 199-203, 1999. PMID: 10660198.

16 Hall EJ and Wuu C: Radiation-induced second cancers: The impact of 3D-CRT and IMRT. Int J Radiat Oncol Biol Phys 56: 83-88, 2003. PMID: 12694826. DOI: 10.1016/s0360-3016(03) 00073-7

17 Kry SF, Salehpour M, Followill DS, Stovall M, Kuban DA, White RA and Rosen II: The calculated risk of fatal secondary malignancies from intensity-modulated radiation therapy. Int $\mathrm{J}$ Radiat Oncol Biol Phys 62: 1195-1203, 2005. PMID: 15990025. DOI: $10.1016 /$ j.ijrobp.2005.03.053

18 Blyth BJ, Kakinuma S, Sunaoshi M, Amasaki Y, Hirano-Sakairi S, Ogawa K, Shirakami A, Shang Y, Tsuruoka C, Nishimura M and Shimada Y: Genetic analysis of $t$ cell lymphomas in carbon ion-irradiated mice reveals frequent interstitial chromosome deletions: implications for second cancer induction in normal tissues during carbon ion radiotherapy. PLoS One 10(6): e0130666, 2015. PMID: 26125582. DOI: 10.1371/journal. pone. 0130666

19 Chung CS, Yock TI, Nelson K, Xu Y, Keating NL and Tarbell $\mathrm{NJ}$ : Incidence of second malignancies among patients treated with proton versus photon radiation. Int J Radiat Oncol Biol Phys 87: 46-52, 2013. PMID: 23778197. DOI: 10.1016/j.ijrobp. 2013.04.030

20 Bekelman JE and Schultheiss T: Berrington de Gonzalez A: Subsequent malignancies after proton versus photon radiation therapy. Int J Radiat Oncol Biol 87: 1-3, 2013. PMID: 23810521. DOI: 10.1016/j.ijrobp.2013.05.016

21 Mohamad O, Tabuchi T, Nitta Y, Nomoto A, Sato A, Kasuya G, Makishima H, Choy H, Yamada S, Morishima T, Tsuji H, Miyashiro I and Kamada T: Risk of subsequent primary cancers after carbon ion radiotherapy, photon radiotherapy, or surgery for localised prostate cancer: a propensity score-weighted, retrospective, cohort study. Lancet Oncol 20: 674-685, 2019. PMID: 30885458. DOI: 10.1016/S1470-2045(18)30931-8

22 Facoetti A, Mariotti L, Ballarini F, Bertolotti A, Nano R, Pasi F, Ranza E and Ottolenghi A: Experimental and theoretical analysis of cytokine release for the study of radiation-induced bystander effect. Int J Radiat Biol 85: 690-699, 2009. PMID: 19637080. DOI: $10.1080 / 09553000903020016$

23 Mancuso M, Pasquali E, Leonardi S, Tanori M, Rebessi S, Di Majo V, Pazzaglia S, Toni MP, Pimpinella M, Covelli V and Saran A: Oncogenic bystander radiation effects in Patched heterozygous mouse cerebellum. Proc Natl Acad Sci USA 105: 12445-12450, 2008. PMID: 18711141. DOI: 10.1073/pnas.080 4186105

24 Rusin A, Lapied E, Le M, Seymour C, Oughton D, Haanes H and Mothersill C: Effect of gamma radiation on the production of bystander signals from three earthworm species irradiated in vivo. Environ Res 168: 211-221, 2019. PMID: 30317106. DOI: 10.1016/j.envres.2018.09.023

25 Kong EY, Yeung WK, Chan TK, Cheng SH and Yu KN: Exogenous nitric oxide suppresses in vivo $\mathrm{X}$-ray-induced targeted and non-targeted effects in Zebrafish embryos. Int J Mol Sci 17, 2016. PMID: 27529238. DOI: 10.3390/ijms17081321

26 Wang T, Xu W, Deng C, Xu S, Li F, Wu Y, Wu L and Bian P: A pivotal role of the jasmonic acid signal pathway in mediating radiation-induced bystander effects in Arabidopsis thaliana. Mutat Res 791-792: 1-9, 2016. PMID: 27497090. DOI: 10.1016/ j.mrfmmm.2016.07.002

27 Vitolo V, Fiore MR, Barcellini A, Vischioni B, Iannalfi A, Facoetti A, Fossati P, Bonora M, Ronchi S, D'Ippolito E, Petrucci R, Viselner G, Ciocca M, Preda L, Valvo F and Orecchia R: Carbon ion radiotherapy in the management of the tumors of the peripheral nervous system. Anticancer Res 39: 909-913, 2019. PMID: 30711975. DOI: 10.21873/anticanres.13193 